\title{
A Spectral Method for Unbounded Flow in a Cylindrical Coordinate System
}

\author{
Xungang Shi and Lixin Wu \\ State Key Laboratory for Turbulence Research \\ Dept. of Mechanics, \\ Peking University, \\ Beijing, 100871, P.R. of China
}

\begin{abstract}
Fourier expansions in the radial direction for unbounded flows expressed in a cylindrical coordinate system are proposed. By appropriate coordinate mapping and periodic extension in the $r$ direction, periodic boundary conditions required by Fourier expansions and infinite differentiability demanded by spectral convergence are established. Appropriate zero factors for the general Fourier expansions are given at the axis and at infinity in order to remove the numerical singularity at $r=0$ and to satisfy all the boundary conditions. The effectiveness of these expansions are demonstrated by the simulation of steady axisymmetrical vortex rings in ideal fluid and the numerical simulation of the head-on collision of two coaxial, equal and opposite vortex rings.
\end{abstract}

\section{Introduction}

Spectral methods have been extensively utilized in large scale computation in meteorology, magnetohydrodynamics and turbulent flows $[2-5,8]$. The key element of spectral methods is the choice of basis functions which depends largely on the boundary condition of the problem. Owing to its simplicity, popularity and fast algorithm, Fourier expansions have been most frequently used as basis functions. However, due to the requirement of periodic boundary conditions, its application range is severely limited.

The choise of basis functions depends also on the coordinate system in which the problem is conveniently described. For flows with some axisymmetry, a cylindrical coordinate system (CCS) is likely most appropriate. In the azimuthal direction, it is natural to choose Fourier series as a basis function. In the radial direction, for bounded flows, Chebychev or Legendre polynomials as well as Bessel functions [4] are possible choices. As for unbounded flows, only Hermite and Laguerre polynomials are applicable for infinite intervals $[0,+\infty)$ and $(-\infty,+\infty)$, respectively. Another approach is to use the above functions for bounded flow after applying a coordinate mapping which transforms the original infinite interval into a finite one. However, for all these orthogonal eigenfunctions, except Chebychev polynomials, no fast algorithm like FFT could be utilized, thus the computations with these basis functions are often inefficient and expensive.

Another difficulty in the numerical computations in CCS is the numerical singularity at $r=0$, due to the fact that some terms of the Navier-Stokes equations in CCS involve factors of the form $r^{-1}$ or $r^{-2}$. However, this singularity is nonphysical and nonintrinsic, because a point on the axis is simply regular interior point of the flow field and where no singularity is described in a Cartesian system. So it is possible to remove this numerical singularity if the asymptotic property of the 
various functions at the axis could be carefully analyzed and appropriate spectral expansions are adopted.

This paper aims at proposing a spectral method for unbounded flow in CCS using Fourier expansions in the radial direction. General Fourier expansions, which could have spectral convergence, are given in order to automatically eliminate the numerical singularity on the axis and to satisfy the boundary conditions at the axis and at infinity. The validity of this method is confirmed by two examples.

\section{Parity and asymptotic properties in CCS}

In the next two sections, all the dependence on the axial coordinate $z$ are temporarily ignored. On the $(r, \theta)$ plane, all functions could be extended to the full plane according to the following rules:

$$
A(-r, \theta)= \pm A(r, \theta+\pi)
$$

in which + is for any scalar $\psi$ and $A_{z}$, while - is for $A_{r}$ and $A_{\theta}$, with $\left(A_{r}, A_{\theta}, A_{z}\right)$ being three components of any vector in CCS.

In general, any flow quantities could be expanded into Fourier series in $\theta$ :

$$
A(r, \theta)=\sum A_{m}(r) \exp (\operatorname{im} \theta)
$$

For an axisymmetric case, $m=0$; for a circumferentially periodic case with wavelength $2 \pi / \beta$, (where $\beta$ must be an integer), $m=n \beta, n$ is any integer.

According to Lewis and Bellan [6], if a function $A$ is infinitely differentiable at $r=0$, then $A_{m}(r)$ could be expanded into a Taylor series in $r$ :

$$
A_{m}(r)=r^{p} \sum_{n=0} a_{m n} r^{2 n}
$$

Various functions have the same parity as the integer $p$, of which the values are shown in Table I, and the order of zeros at $r=0$ is not less than $p$.

Now, let's discuss some asymptotic properties at infinity. For vortical flows, vorticity is usually confined in a finite spatial region. In other words, the flow far enough from the origin could be considered as an inviscid potential flow, which might be thought to be induced by some sources distributed in a finite spatial region and the total amount of sources being zero. The fluid at infinity is either still or moving with a uniform axial velocity. It could be shown that any flow quantities after deducting the uniform flow have the following asymptotic expansion as $r \rightarrow \infty$, provided that it is infinitely differentiable at infinity, (see appendix):

$$
A_{m}(r)=r^{-q} \sum_{n=0} b_{m n} r^{-2 n}
$$

in which $q$ is an integer given also in Table I, and the order of zeros at infinity is not less than $q$.

\section{Radial Fourier expansions for unbounded flow}

Firstly, in order to meet the basic requirement of Fourier expansions, a trigonometrical mapping in the radial direction is introduced,

$$
r=L \tan \frac{\eta}{2}
$$


Table I: Parity of functions and order of zeros at $r=0$ and $\infty$.

\begin{tabular}{|c|c|c|c|c|}
\hline func- & $\psi$ & $A_{z m}$ & \multicolumn{2}{|c|}{$A_{r m}, A_{\theta m}$} \\
\cline { 4 - 5 } tion & & & $m=0$ & $m \neq 0$ \\
\hline$p$ & $|m|$ & $|m|$ & 1 & $|m|-1$ \\
\hline$q$ & $|m|+1$ & $|m|+3$ & 4 & $|m|+2$ \\
\hline
\end{tabular}

which transforms the original infinite interval $-\infty<r<+\infty$ into a finite one $-\pi<\eta<+\pi$. At the same time, the transformed N-S equation system involves only periodic coefficients with wavelength $2 \pi$ and consequently permits periodic solutions to exist. The same mapping has been used by Cain et al. [1] and the present authors [13] for time developing planar mixing layers.

After the mapping (4), if $\phi(\eta)$ represents anyone of $A_{m}(\eta)$, as $\eta \rightarrow \pm \pi$, we have:

$$
\begin{array}{ll}
\phi(\pi) & =\phi(-\pi)=0 \\
\frac{d \phi}{d \eta} & =\frac{d \phi}{d r} \frac{d r}{d \eta}=O\left(r^{-q-1}\right) \cdot \frac{L}{2}\left[1+\left(\frac{r}{L}\right)^{2}\right]=O\left(r^{1-q}\right)
\end{array}
$$

Then, so long as $q>1$, we have

$$
\phi^{\prime}(\pi)=\phi^{\prime}(-\pi)=0
$$

Provided that the original function $\phi(r)$ is in the $C_{1}$ class, function $\phi(\eta)$ could always be periodically extended outside $\eta= \pm \pi$ with wavelength $2 \pi$ and might be expanded as

$$
\begin{array}{ll}
\phi=\sum a_{n} \cos n \eta & \text { for even functions } \\
\phi=\sum b_{n} \sin n \eta & \text { for odd functions }
\end{array}
$$

However, owing to the singularity of the mapping (4) at $\eta= \pm \pi$, differentiability of the extended function $\phi(\eta)$ might be impaired there,

$$
\begin{array}{ll}
\frac{d^{j} \phi}{d \eta^{j}}=\frac{d}{d r}\left[\frac{d^{j-1} \phi}{d \eta^{j-1}}\right] \cdot \frac{d r}{d \eta}=O\left(r^{j-q}\right) \rightarrow 0 & j<q \\
\frac{d^{q+2 j} \phi}{d \eta^{q+2 j}}=\sum_{n=0} c_{n} r^{-2 n} \rightarrow c_{0} \neq 0 & j \geq 0 \\
\frac{d^{q+2 j+1} \phi}{d \eta^{q+2 j+1}}=O\left(r^{-1}\right) \rightarrow 0 & j \geq 0
\end{array}
$$

As a result of (6), only if the extension is even for function $\frac{d^{q+2 j} \phi}{d \eta^{q+2 j}}$ with respect to $\eta= \pm \pi$, namely, only if the extension for function $\phi(\eta)$ has the same parity as the integer $q$, the extended function $\phi(\eta)$ could be infinitely differentiable on the whole real axis, i.e. being in the $C_{\infty}$ class, provided that the original function $\phi(r)$ is also in the $C_{\infty}$ class. From Table I it is noted that the parity of function $\phi$ is always opposite to that of integer $q$ for all cases, that means that the extension of $\phi$ with respect to $\eta= \pm \pi$ must be even for odd functions, or odd for even functions and always having a wavelength of $4 \pi$. Then the Fourier expansions of the extended functions have spectral convergence and could be expressed as the following forms:

$$
\begin{array}{ll}
\phi=\sum a_{n} \cos \left(n+\frac{1}{2}\right) \eta & \text { for even functions } \\
\phi=\sum b_{n} \sin \left(n+\frac{1}{2}\right) \eta & \text { for odd functions }
\end{array}
$$

Otherwise, if one uses the Fourier expansions (5) with wavelength $2 \pi$, the values of $\frac{d^{q+2 j} \phi}{d \eta^{q+2 j}}$ at $\eta= \pm \pi$ are necessarily discontinuous for $j \geq 0$, the function $\phi(\eta)$ is in the $C_{q-1}$ class, the expansions will have convergence accuracy of only order $q-1$. 
Table II: Selected forms of function $g(\psi)$ when $\psi \geq F \geq 0$.

\begin{tabular}{|c|c|c|c|c|c|}
\hline forms & uniform & step & linear & parabolic & exponential \\
\hline$g(\psi)=$ & $B$ & $\begin{array}{l}A, \quad \psi \geq F_{1} \\
B, \quad F \leq \psi<F_{1}\end{array}$ & $B(\psi-F)$ & $B(\psi-F)^{2}$ & $B\left(e^{\psi}-e^{F}\right)$ \\
\hline
\end{tabular}

Because some terms appearing in the N-S equation system in CCS involve factors of the form $r^{-1}$ or $r^{-2}$, numerical singularity might occur at $r=0$. In order to remove this singularity, a zerofactor of order 2 at $\eta=0$ would best be separated from the expansions beforehand. Separating a similar zero-factor at $\eta= \pm \pi$ simultaneously is also allowable (if $q>1$ ) and helpful for satisfying the boundary condition at infinity. Then the Fourier expansions could be rewritten into the following forms for even or odd functions, respectively:

$$
\begin{aligned}
& \phi=\frac{\phi_{0}}{4}\left(3 \cos \frac{\eta}{2}+\cos \frac{3 \eta}{2}\right)+(1-\cos 2 \eta) \sum a_{n} \cos \left(n+\frac{1}{2}\right) \eta \\
& \phi=\phi_{0}^{\prime} \sin \eta \cos \frac{\eta}{2}+(1-\cos 2 \eta) \sum b_{n} \sin \left(n+\frac{1}{2}\right) \eta
\end{aligned}
$$

\section{Examples}

\subsection{Steady axisymmetric solutions of vortex ring in ideal fluid [12]}

For this case, $\omega / r=g(\psi)$ is an arbitrarily given function of streamfunction $\psi$. Steady motion could exist only if the reference system moves with the self-induced translational speed $W$, which depends on the function $g(\psi)$ and shall be determined together with the solution. There will be a uniform flow of speed $-W$ at infinity. After deducting the uniform flow, the streamfunction $\psi$ should satisfy the following equation and boundary conditions:

$$
\begin{array}{ll}
\frac{\partial^{2} \psi}{\partial r^{2}}-\frac{1}{r} \frac{\partial \psi}{\partial r}+\frac{\partial^{2} \psi}{\partial z^{2}}= & -r^{2} g\left(\psi-\frac{1}{2} W r^{2}\right) \\
\psi=0, & \text { as } r \rightarrow 0 \text { and } \infty
\end{array}
$$

To solve this problem, an iterative procedure is proposed, in which, for each iteration $\psi(r, z)$ and $W$ in the right hand side of the equation are taken from the result of the last iteration and $\psi$ is expanded as

$$
\psi=(1-\cos \eta) \sum_{k=-K}^{K} \sum_{n=0}^{N} a_{n k} \cos \left(n+\frac{1}{2}\right) \eta \exp (i k \gamma z)
$$

where $\gamma$ is an axial wavenumber, which means that an axially periodic array of vortex rings is assumed. Noting that the streamfunction induced by a vortex ring has zeros of only order one at infinity, i.e. $q=1$, only zero-factor at $\eta=0$ is separated from the expansion. This expansion has spectral convergence.

Five types of function $g(\psi)$ were selected to search for solutions. They all are equal to zero when $\psi<F$, and as $\psi \geq F \geq 0$, have the forms listed in Table II, in which, $A>B>0,0 \leq F<F_{1} \leq 1$, are adjustable constant parameters. For all these cases, steady solutions are readily obtained. Figure 1 shows the streamline patterns for uniform $g(\psi),(\mathrm{a}),(\mathrm{b})$ and (c) correspond to $F=0.7$, 0.4 and 0 , or relative core radius $\alpha=a / R=0.15,0.47$ and $\sqrt{2}$, respectively. The last one is just the famous Hill's spherical vortex. The black parts in (a) and (b) are regions of vortex core where $g \neq 0$. The resolution used in the calculations is $K=N=121$. 


\subsection{Numerical simulation of head-on collision of two coaxial equal and opposite vortex rings [11]}

The same problem was studied by Stanaway et al. [14] in a spherical polar coordinate system. The basis functions are composed of Legendre polynomials in the polar direction and Jacobi polynomials matched to an algebraic mapping of the radial coordinate. Now we treat it in CCS. The N-S equation system for axisymmetric flows in terms of vorticity and streamfunction can be written as

$$
\begin{aligned}
& \left(\frac{\partial}{\partial t}+v_{r} \frac{\partial}{\partial r}+v_{z} \frac{\partial}{\partial z}\right) g=\nu\left(\frac{\partial^{2}}{\partial r^{2}}+\frac{3}{r} \frac{\partial}{\partial r}+\frac{\partial^{2}}{\partial z^{2}}\right) g \\
& \left(\frac{\partial^{2}}{\partial r^{2}}-\frac{1}{r} \frac{\partial}{\partial r}+\frac{\partial^{2}}{\partial z^{2}}\right) \psi=-r^{2} g
\end{aligned}
$$

Close examination shows that for this problem the streamfunction $\psi$ has zeros of order 2 at $r=0$ and order 3 as $r \rightarrow \infty$ and the function $g$ is finite on the axis and identically vanishing when $r$ is large enough.

For this example the adopted Fourier expansions are shown as follows,

$$
\begin{aligned}
\psi & =(1-\cos 2 \eta) \sum_{k} \sum_{n} \psi_{n k} \cos n \eta \exp (i k \gamma z) \\
g & =\sum_{k}\left[g_{k} \cos ^{2} \frac{\eta}{2}+(1-\cos 2 \eta) \sum_{n} g_{n k} \cos n \eta\right] \exp (i k \gamma z)
\end{aligned}
$$

Because these expansions are of the form of (5) having wavelength of $2 \pi$, the expansion for $\psi$ has convergence accuracy of only finite order. It is believed that if expansions of form (8) are adopted, the accuracy could be improved.

Figure 2 displays the contours of $g$ during the process of collision. It can be seen that the circulation of each vortex ring decreases quickly since two vortex rings contact each other due to viscous annihilation across the collision plane. At the final stage, the core area evolves into a pupalike head-tail structure, which was also observed in the simulation of two anti-parallel vortex tubes by Melander and Hussain [7]. Parameters are chosen as $R e=\Gamma_{0} / \nu=772$, initial relative core radius $\alpha_{0}=0.35$, initial relative distance $2 Z_{0} / R_{0}=3.0$, resolution is $K \times N=40 \times 121$, $\triangle t=0.001$.

\section{Conclusion}

We proposed a spectral method for unbounded flow in CCS using Fourier expansions in the radial direction instead of other orthogonal eigenfunctions. Its main advantage lies in three aspects. First, complicated calculation involved in other orthogonal eigenfunctions is avoided and the fast algorithm FFT can be utilized. Higher efficiency and higher accuracy could be obtained. Second, the numerical singularity occurring at the axis could be removed. Third, the boundary conditions at $r=0$ and $r \rightarrow \infty$ are already satisfied. The validity of this method was demonstrated by the above two examples. More examples for non-axisymmetrical flows could be found in [9] and [10]. The prospect of potential application of this method might be great.

\section{Acknowledgements}

This project is supported by the National Natural Science Foundation of China and the Doctoral Program of the Institution of Higher Education of China. 


\section{References}

[1] Cain, A.B., Reynolds, W.C., and Ferziger, J.H., Thermosci. Div. Rep. TF-14, Dept. of Mech. Eng., Stanford Univ., 1981.

[2] Canuto, C., Hussaini, M.Y., Quarteroni, A. and Zang, T.A., Spectral Methods in Fluid Dynamics, Springer-Verlag, 1987.

[3] Chen, S. and Shan, X., Comp. Phys., 6, pp. 643, 1992.

[4] Chen, H., Shan, X. and Montgomery, D., Phys. Rev. A, 42, pp. 6158-6165, 1990.

[5] Gottlieb, D. and Orszag, S.A., Numerical Analysis of Spectral Methods, NSF-CMBS Monograph No. 26, SIAM, Philadelphia, PA, 1977.

[6] Lewis, H.R. and Bellan, P.M., J. Math. Phys., 31, pp. 2592, 1990.

[7] Melander, M.V. and Hussain, F., in CTR Proc. 1988 Summer Program, pp. 257, 1998.

[8] Melander, M.V. and Zabusky, N.J., Fluid Dyn. Res., 3, pp. 247-250, 1988.

[9] Shi, X.G. and Wu, L.X., "Circumferential Instability of Vortex Rings," Proc. 6th Asian Congress of Fluid Mechanics, Singapore, 2, pp. 1210-1214, 1995.

[10] Shi, X.G. and Wu, L.X., "A Spectral Method for Unbounded Flows in a Cylindrical Coordinate System," Proc. 1st Asian Comp. Fluid Dyn. Conf., Hong Kong, 2, pp. 441-446, 1995.

[11] Shi, X.G. and Wu, L.X., in Proc. 2nd Int'l. Conf. on Fluid Mech., Beijing, edited by F.G. Zhuang, Peking Univ. Press, China, pp. 521-526, 1993.

[12] Shi, X.G. and Wu, L.X., in Proc. 2nd Int'l. Conf. on Fluid Mech., Beijing, edited by F.G. Zhuang, Peking Univ. Press, China, pp. 527-532, 1993.

[13] Shi, X.G. and Wu, L.X., in Some New Trends on Fluid Mechanics and Theoretical Physics (ICFMTP, 1992), edited by C.C. Lin, Peking Univ. Press, China, pp. 191-200, 1992.

[14] Stanaway, S.K., Cantwell, B.J. and Spalart, P.R., AIAA Paper 88-0318, 1988.

\section{Appendix}

\section{Asymptotic properties of various functions as $r \rightarrow \infty$}

Any flow field far enough from the origin could be viewed as a potential flow induced by some sources distributed in a finite spatial region and the total amount of sources being zero. In general, the velocity potential function in CCS could be expressed as

$$
\phi(r, \theta, z)=-\frac{1}{4 \pi} \sum_{j=1}^{N} \frac{q_{j}}{R_{j}}
$$

where $q_{j}$ is the strength of the $j$-th point source, of which the total amount must satisfy $\sum_{j=0}^{N} q_{j}=0$, $R_{j}=\left[r^{2}-2 r r_{j} \cos \left(\theta-\theta_{j}\right)+r_{j}^{2}+\left(z-z_{j}\right)^{2}\right]^{1 / 2}$ is the distance from any point $(r, \theta, z)$ in space to the 
point $\left(r_{j}, \theta_{j}, z_{j}\right)$ where the $j$-th point source is located. The term containing cos could be rewritten as

$$
2 r_{j} \cos \left(\theta-\theta_{j}\right)=r_{j}\left[e^{i\left(\theta-\theta_{j}\right)}+e^{-i\left(\theta-\theta_{j}\right)}\right]=\zeta_{j}^{*} e^{i \theta}+\zeta_{j} e^{-i \theta}
$$

in which, $\zeta_{j}=r_{j} e^{i \theta_{j}}$ and $\zeta_{j}^{*}$ is its complex conjugate. Then three components of velocity vector could be obtained:

$$
\begin{aligned}
& v_{r}=\frac{\partial \phi}{\partial r}=\frac{1}{4 \pi} \sum_{j} \frac{q_{j}}{R_{j}^{3}}\left[r-\frac{1}{2}\left(\zeta_{j}^{*} e^{i \theta}+\zeta_{j} e^{-i \theta}\right)\right] \\
& v_{\theta}=\frac{1}{r} \frac{\partial \phi}{\partial \theta}=\frac{i}{8 \pi} \sum_{j} \frac{q_{j}}{R_{j}^{3}}\left(\zeta_{j} e^{-i \theta}-\zeta_{j}^{*} e^{i \theta}\right) \\
& v_{z}=\frac{\partial \phi}{\partial z}=\frac{1}{4 \pi} \sum_{j} \frac{q_{j}}{R_{j}^{3}}\left(z-z_{j}\right)
\end{aligned}
$$

As $r \rightarrow \infty, R_{j}^{-3}$ has the following asymptotic expansion:

$$
\begin{aligned}
\frac{1}{R_{j}^{3}} & =\frac{1}{r^{3}}\left\{1-\frac{\zeta_{j}^{*} e^{i \theta}+\zeta_{j} e^{-i \theta}}{r}+\frac{r_{j}^{2}+\left(z-z_{j}\right)^{2}}{r^{2}}\right\}^{-3 / 2} \\
& =\frac{1}{r^{3}}\left\{1+\sum_{k=1} \frac{(2 k+1) ! !}{2^{k} k !}\left\{\frac{\zeta_{j}^{*} e^{i \theta}+\zeta_{j} e^{-i \theta}}{r}-\frac{r_{j}^{2}+\left(z-z_{j}\right)^{2}}{r^{2}}\right\}^{k}\right\} \\
& =\sum_{m} \frac{e^{i m \theta}}{r^{|m|+3}} \sum_{n=0} \frac{a_{m n}^{(j)}}{r^{2 n}}
\end{aligned}
$$

in which the coefficients $a_{m n}^{(j)}$ could depend on the coordinate $z$ and $a_{00}^{(j)}=1$, for all $j$.

Substituting this expansion into the expressions of velocity components, noting that $\sum_{j=1}^{N} q_{j}=0$ and $a_{00}^{(j)}=1$, the asymptotic expansions of velocity components could be obtained:

$$
\begin{aligned}
& v_{r}=\frac{1}{r^{4}} \sum_{n=0} \frac{b_{0 n}}{r^{2 n}}+\sum_{m \neq 0} \frac{e^{i m \theta}}{r^{|m|+2}} \sum_{n=0} \frac{b_{m n}}{r^{2 n}} \\
& v_{z}=\sum_{m} \frac{e^{i m \theta}}{r^{|m|+3}} \sum_{n=0} \frac{c_{m n}}{r^{2 n}}
\end{aligned}
$$

$v_{\theta}$ has the same form as $v_{r}$. But for the vorticity vector, all three components are identically equal to zero if $r$ is large enough.

As for scalar functions, based on the same argument, the velocity potential could be expanded as

$$
\phi=\frac{1}{r^{3}} \sum_{n=0} \frac{d_{0 n}}{r^{2 n}}+\sum_{m \neq 0} \frac{e^{i m \theta}}{r^{|m|+1}} \sum_{n=0} \frac{d_{m n}}{r^{2 n}}
$$

However, potential functions can not be used to describe whole flow fields involving vorticity. For axisymmetrical flows, $m=0$, there is a scalar streamfunction $\psi$ which could express axisymmetrical vortex flows and could be expanded as

$$
\psi=\frac{1}{r} \sum_{n=0} \frac{f_{n}}{r^{2 n}}
$$

Therefore we have the values of the integer $q$ shown in Table I. 


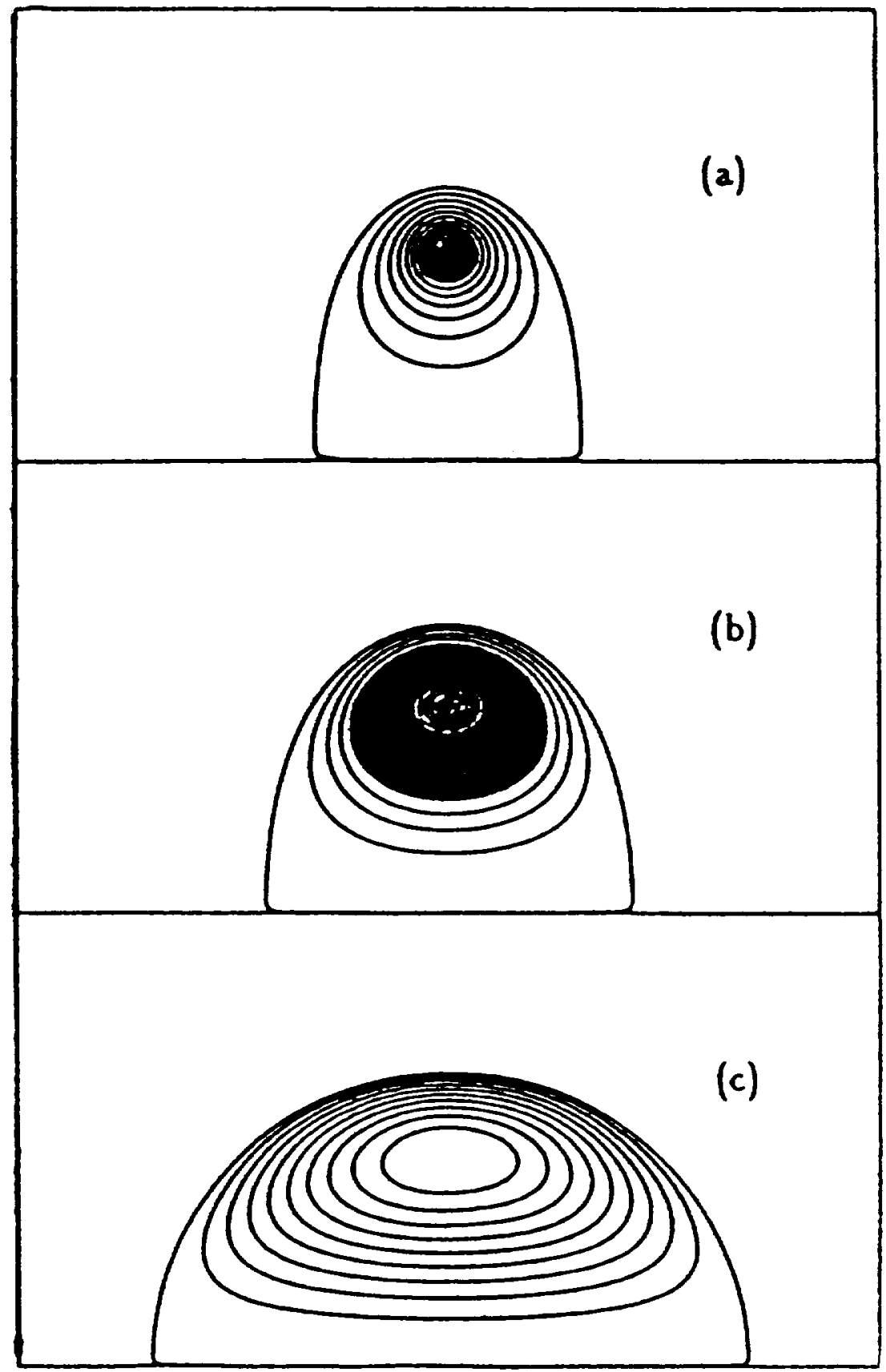

Figure 1: Streamline patterns for $g(\psi)=$ Const. Parameter $\alpha$ is (a) 0.15 , (b) 0.47 and (c) $\sqrt{2}$, respectively. 

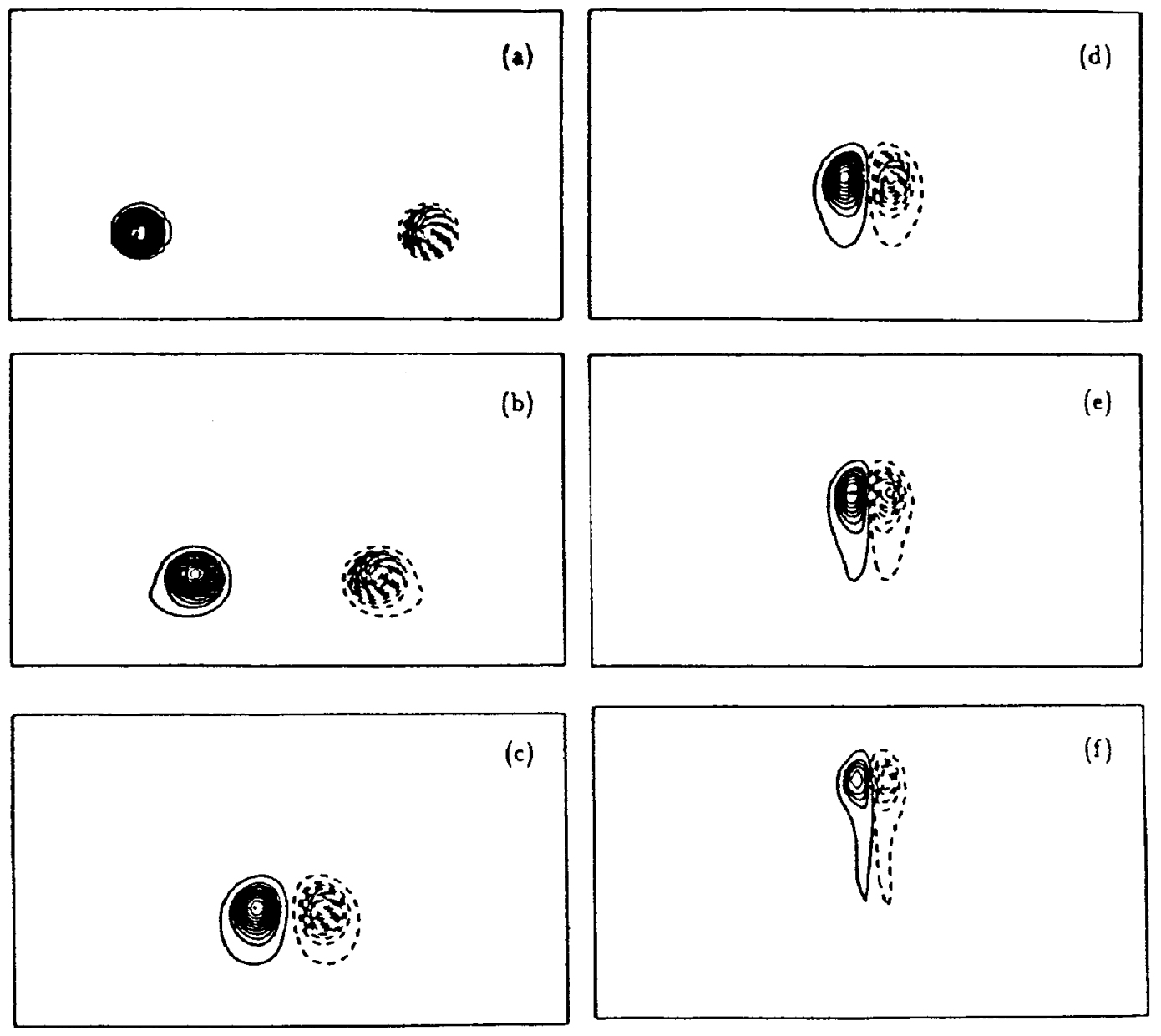

Figure 2: Temporal evolution of contours of $g$ during collision. The corresponding times are (a) 0.0, (b) 1.0, (c) 2.0, (d) 3.0, (e) 4.0, (f) 5.0. $R e=\Gamma_{0} / \nu=772, \alpha_{0}=0.35, x_{0} / R_{0}=3$. 